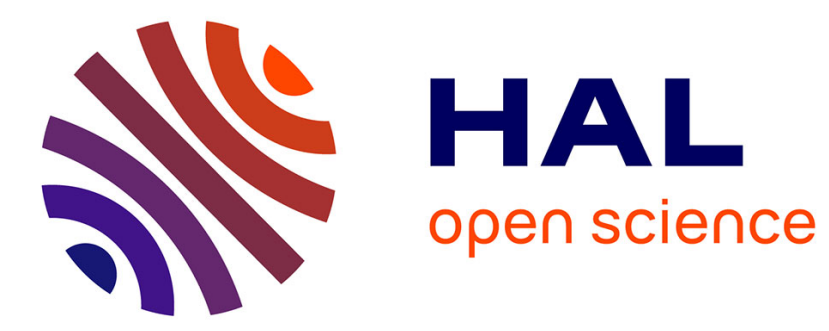

\title{
Shape, displacement and mechanical properties from isogeometric multiview stereocorrelation
}

\author{
John-Eric Dufour, François Hild, Stéphane Roux
}

\section{To cite this version:}

John-Eric Dufour, François Hild, Stéphane Roux. Shape, displacement and mechanical properties from isogeometric multiview stereocorrelation. The Journal of Strain Analysis for Engineering Design, 2015, 50 (7), pp.470-487. 10.1177/0309324715592530 . hal-01198921

\section{HAL Id: hal-01198921 \\ https://hal.science/hal-01198921}

Submitted on 14 Sep 2015

HAL is a multi-disciplinary open access archive for the deposit and dissemination of scientific research documents, whether they are published or not. The documents may come from teaching and research institutions in France or abroad, or from public or private research centers.
L'archive ouverte pluridisciplinaire HAL, est destinée au dépôt et à la diffusion de documents scientifiques de niveau recherche, publiés ou non, émanant des établissements d'enseignement et de recherche français ou étrangers, des laboratoires publics ou privés. 


\title{
Shape, Displacement and Mechanical Properties from Isogeometric Multiview
}

\author{
Stereocorrelation
}

John-Eric Dufour, ${ }^{1,2}$ François Hild, ${ }^{2, *}$ and Stéphane Roux ${ }^{2}$

${ }^{1}$ SAFRAN Snecma Villaroche

Rond-point René Ravaud, 77550 Reau, France

${ }^{2}$ LMT, ENS Cachan/CNRS/Université Paris Saclay

61 avenue du Président Wilson, F-94235 Cachan Cedex, France

\begin{abstract}
It is proposed to develop a multiview framework to perform stereocorrelation by resorting to CAD-based descriptions of the observed 3D surfaces. Once the 3D surfaces have been reconstructed, the corresponding 3D displacement fields are measured within the same space. These fields are subsequently utilized to update isogeometric analyses to calibrate the elastic properties in a tensile experiment. An alternative route consists of preforming such analyses within an integrated framework.
\end{abstract}

\footnotetext{
*Corresponding author, email: hild@lmt.ens-cachan.fr
} 
Keywords: 3D surfaces; Identification; Isogeometric analyses; Kinematic measurements; NURBS; Stereocorrelation.

\section{Introduction}

Digital Image Correlation (DIC) has reached a state of development that makes it possible to create new bridges between experiments and simulations [33]. To mention but one example, finite-element based DIC procedures were proposed ten years ago $[5,32,4]$ and have been used in various applications since then [22]. Similarly, new tools are currently implemented to integrate Computer-Aided Design (CAD) and Finite Element Analyses (FEA) [9]. Recently, stereocorrelation approaches have been developed using CAD descriptions of the observed surface [2]; FE-based surfaces can also be analyzed [13]. 'Linking CAD tools, FE simulations and DIC procedures is (thus) becoming possible, [34].

One area of research that needs strong links between experiments and simulations is the identification and validation of numerical material models. For instance, finite element model updating (FEMU) was introduced very early on to determine elastic parameters [24, 23, 7]. Since the early developments of FEMU, other methods have been proposed, some of them explicitly requiring full-field measurements $[1,18]$. In all these approaches, the measurement and identification steps are performed independently and sequentially. An alternative route consists of performing these two steps in an integrated way by either using closed-form solutions (e.g., Brazilian test [20], cracks in elastic media [29]) or numerically generated sensitivity 
fields [25, 31, 26].

In the following, it is proposed to extend all these concepts to isogeometric analyses. The reasons for using the latter ones are two-fold. First, CAD modelers are commonly used in mechanical design and therefore a vast majority of fabricated structures is known virtually via its nominal shape. This is a very precious piece of information that is seldom used in DIC procedures [2]. Second, with the development of isogeometric analysis (IGA) frameworks, the other end of the virtual world, namely, mechanical sizing via finite element analyses can be integrated into conventional NURBS-based CAD design tools [28]. In the experiment / CAD / IGA triptych, the missing link is on the experimental side. The aim of the present paper is to show that it is possible to reconstruct 3D shapes, measure 3D displacement fields and calibrate mechanical properties by resorting to isogeometric stereocorrelation.

In order to observe the whole external surfaces of a structure, a multiview framework is first introduced within a global stereocorrelation setting. The different steps associated with stereocorrelation are discussed in Section 2, namely, calibration, measurement of 3D shapes and 3D displacements. This is all the more true as a model shape is used while the texture (gray levels) is only known from the images. Section 3 is devoted to a discussion of the proposed metric. A proof of concept is then proposed in Section 4 to validate all the various implementations. It consists of performing a tensile test on a squared bar monitored with 4 digital single-lens reflex cameras. From these first analyses, the complete 3D shape is reconstructed and the 3D displacement fields during the mechanical test are measured. With these data, Section 5 shows that an IsoGeometric Model Updating (IGMU) pro- 
cedure can be designed to extract elastic constants. Last, a fully integrated isogeometric stereocorrelation approach is also implemented and presented in Section 6.

\section{Multiview system}

The description of a specimen that is used in this study is a CAD model. The external surface of the solid consists of a collection of $n_{s}$ elementary surfaces. Each of the latter ones $\mathrm{S}_{s}$, which is labeled by $s=1, \ldots, n_{s}$, is described in the Non-Uniform Rational B-Spline (NURBS) framework (recalled in Appendix A) where the three-dimensional shape $\boldsymbol{X}=(X, Y, Z)$ is written as $\boldsymbol{X}\left(\boldsymbol{\xi}^{s}\right)$, where $\boldsymbol{\xi}^{s}=\left(u^{s}, v^{s}\right)$ define the parametric space, conventionally spanning the elementary square $[0,1]^{2}$. Let us emphasize that what is assumed to be known from the CAD model is the virtual (or nominal) shape of the object. In practice, these surfaces will be decorated by a specific texture (i.e., classically a random speckle pattern is painted on the surface) that is not known from the CAD model.

A number $n_{c}$ of cameras is used to observe the specimen as schematically shown in Figure 1. Each camera $C^{i}$ captures an image $f^{i}\left(\boldsymbol{x}^{i}\right)$ where $\boldsymbol{x}^{i}$ is a point in the (two-dimensional) $i$-th image plane.

Stereovision consists of registering the different images taking into account a projection model in order to measure the specimen shape. The projection model itself has first to be identified and its parameters evaluated from a "calibration step" detailed below. The same strategy can be further used to track the change of $3 \mathrm{D}$ shape when the specimen is used in a me- 


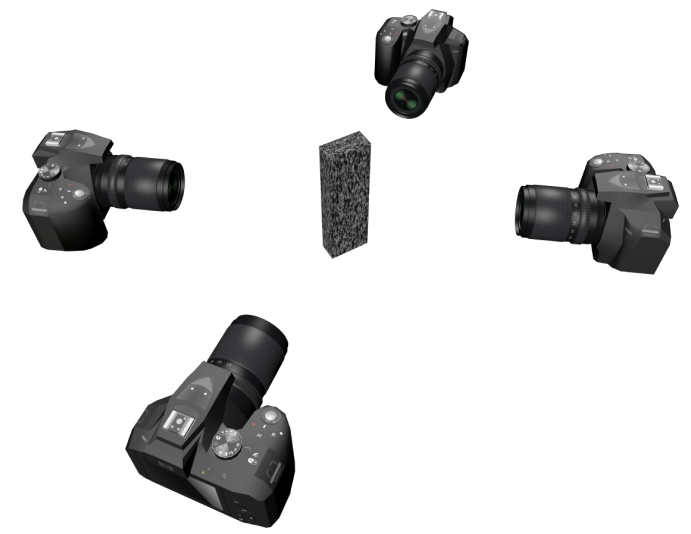

Figure 1: Schematic drawing of a stereovision setup with a multisurface object (parallelepiped) in the center observed by a four-camera system. This elementary example will be used in Section 4 to illustrate the proposed methodology.

chanical test. This amounts to measuring the 3D displacement of the object surface. In the present case, stereocorrelation, which combines stereovision and image correlation, is used as the matching algorithm to measure 3D shapes and 3D displacement fields.

\subsection{Calibration of a multiview system}

The calibration of the multicamera system is achieved by using a global approach to stereocorrelation [2]. In the present case, the object of interest will serve as calibration target since its nominal shape is known. Two formulations of the problem will be introduced below. The first one is based on camera pairs observing a common surface $\mathrm{S}_{s}$. The second one will be used for the registration of each camera image onto a reference one, which is defined in the parametric space and which will be progressively refined. In 
the present paper, only the second approach will be followed, the first one having already been reported and validated for two cameras [2, 12, 11].

First, the projection matrices $[14,35]$ for each camera $\mathrm{C}^{i}$ (i.e., $\left[\boldsymbol{M}^{i}\right]$, which is a $3 \times 4$ matrix) are calibrated by resorting to integrated DIC [20, 29]. The homogeneous coordinates of any $3 \mathrm{D}$ point $\{\overline{\boldsymbol{X}}\}=(X, Y, Z, 1)^{t}$ are related to the corresponding homogeneous coordinates in each camera plane $\left\{\overline{\boldsymbol{x}^{i}}\right\}=$ $\left(\sigma^{i} x^{i}, \sigma^{i} y^{i}, \sigma^{i}\right)^{t}$ by the projection matrix [15]

$$
\left\{\overline{\boldsymbol{x}^{i}}\right\}=\left[\boldsymbol{M}^{i}\right]\{\overline{\boldsymbol{X}}\}
$$

where $\sigma^{i}$ is a scale factor. Reverting to the description of each surface $\mathrm{S}_{s}$ based on the intrinsic coordinate system $\boldsymbol{\xi}^{s}$, the points on the actual surface are further written $\boldsymbol{X}\left(\boldsymbol{\xi}^{s}\right)$. These different elements, namely, projection matrix, scale factor and surface parameterization, allow the position in the image plane $i$ to be expressed for any point parameterized by $\boldsymbol{\xi}^{s}$, and is written $\boldsymbol{x}^{i}\left(\left[\boldsymbol{M}^{i}\right], \boldsymbol{X}\left(\boldsymbol{\xi}^{s}\right)\right)$. The calibration consists of minimizing the sum of squared differences (Figure 2)

$$
\eta^{2}\left(\left[\boldsymbol{M}^{k=1, n_{c}}\right]\right)=\sum_{s=1}^{n_{s}} \sum_{i=1}^{n_{c}-1} \sum_{j>i}\left\|f^{i}\left(\boldsymbol{x}^{i}\left(\left[\boldsymbol{M}^{i}\right], \boldsymbol{X}\left(\boldsymbol{\xi}^{s}\right)\right)\right)-f^{j}\left(\boldsymbol{x}^{j}\left(\left[\boldsymbol{M}^{j}\right], \boldsymbol{X}\left(\boldsymbol{\xi}^{s}\right)\right)\right)\right\|^{2}
$$

with respect to the each unknown matrix $\left[\boldsymbol{M}^{k}\right]$, where $f^{i, j}$ are the image pairs in the reference configuration. The previous minimization is achieved by resorting to Newton-Raphson's method in which linearizations and corrections are performed [22]. The precise expression of the linearized equations to be solved will depend on the details of the expression of the norm $\|\cdots\|$ used in the weighting of residuals. The latter will be discussed in Section 3 . 
To initialize the code, the user has to chose at least 6 remarkable points in each considered picture.

In the present setting, modifying the projection matrix $\left[\boldsymbol{M}^{i}\right]$ by small increments $\delta\left[\boldsymbol{M}^{i}\right]$ induces a motion of the corresponding point from $\boldsymbol{x}^{i}$ to $\boldsymbol{x}^{i}+\delta \boldsymbol{x}^{i}$. The quantity $\delta \boldsymbol{x}^{i}$ is not a physical displacement, but rather the sensitivity with respect to the parameters of the geometrical projection to be determined here, $\{\boldsymbol{\delta} \boldsymbol{m}\}$ namely 11 per camera out of the 12 matrix components

$$
\left\{\boldsymbol{\delta} \boldsymbol{x}^{i}\right\}=\frac{\partial \boldsymbol{x}^{i}}{\partial\left[\boldsymbol{M}^{i}\right]}\left(\left[\boldsymbol{M}^{i}\right], \boldsymbol{X}\right) \delta\left[\boldsymbol{M}^{i}\right] \equiv\left[\boldsymbol{S}_{\boldsymbol{M}}\right]\{\boldsymbol{\delta} \boldsymbol{m}\}
$$

where $\left[\boldsymbol{S}_{\boldsymbol{M}}\right]$ gathers the set of $11 \times n_{c}$ sensitivity fields. The remaining unknowns (one per camera) have to be determined from the knowledge of absolute dimensions in the observed structure [2].

It is worth noting that the sum appearing in Equation (2) is implicitly restricted to cameras $\mathrm{C}^{i}$ and $\mathrm{C}^{j}$ that both can see the surface $\mathrm{S}_{s}$. In practice, considering all pairs of cameras may be demanding, although it would be required in order to reach the best possible determination of the projection matrices $\left[\boldsymbol{M}^{i}\right]$. A decimation is possible to reduce this number down to $n^{c}-1$ camera pairs at most. A different formulation proposed below will be preferred but it requires first an approximate registration to initialize the formulation. Hence, even if the choice of camera pairs is not optimal, it could be first used to obtain a first determination of the projection matrices $\left[\boldsymbol{M}^{i}\right]$.

The second proposed formulation, illustrated in Figure 3 circumvents the problem of having to accumulate all possible camera pairs. The main idea is to perform an individual determination of the camera projection matrix 


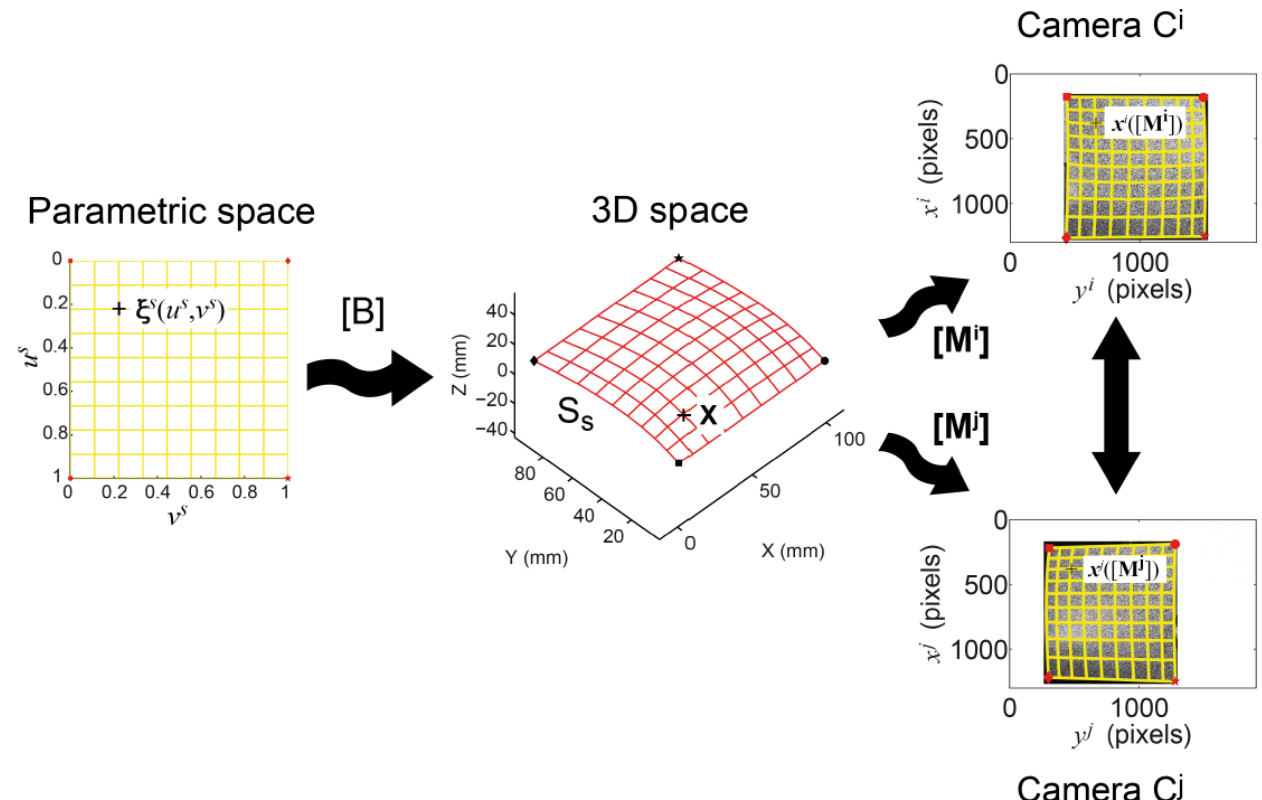

Figure 2: Determination of the projection matrices via global stereocorrelation for a given pair of cameras $\mathrm{C}^{i}-\mathrm{C}^{j}$ and surface $\mathrm{S}_{s}$ defined via its parametric space

$\left[\boldsymbol{M}^{i}\right]$ considering all cameras $\mathrm{C}^{i}$, one after the other, from the registration of the images $f^{i}\left(\boldsymbol{x}^{i}\right)$ onto a reference $\hat{f}\left(\boldsymbol{\xi}^{s}\right)$, which will be referred to as intrinsic texture in the sequel. As briefly discussed in the introduction, it is assumed that the model shape is perfectly known $\boldsymbol{X}(\boldsymbol{\xi})$ although the intrinsic texture is unknown. Were it be known, then the minimization of the following functional

$$
\varrho^{2}\left(\left[\boldsymbol{M}^{k=1, n_{c}}\right]\right)=\sum_{s=1}^{n_{s}} \sum_{i=1}^{n_{c}}\left\|f^{i}\left(\boldsymbol{x}^{i}\left(\left[\boldsymbol{M}^{i}\right], \boldsymbol{X}\left(\boldsymbol{\xi}^{s}\right)\right)\right)-\hat{f}\left(\boldsymbol{\xi}^{s}\right)\right\|^{2}
$$

with respect to all $\left[\boldsymbol{M}^{k}\right]$ matrices would deliver an ideal calibration step. However, if an (even approximate) determination of the projection matrices $\left[\boldsymbol{M}^{i}\right]$ is known, each image point $\boldsymbol{x}^{i}$ can be mapped onto a $\boldsymbol{X}\left(\boldsymbol{\xi}^{s}\right)$ point on 
surface $\mathrm{S}_{s}$, and hence, averaging over all images observing the same physical surface (possibly after correction) provides such a reference object with its intrinsic texture

$$
\hat{f}\left(\boldsymbol{\xi}^{s}\right)=\left\langle f^{i}\left(\boldsymbol{x}^{i}\left(\left[\boldsymbol{M}^{i}\right], \boldsymbol{X}\left(\boldsymbol{\xi}^{s}\right)\right)\right)\right\rangle_{i}
$$

where $\langle\cdots\rangle$ denote averages. In Section 3, possible corrections of $f^{i}$ (e.g., modification of gray levels, spatial filtering) will be discussed in more details. This writing assumes that a gray level is an absolute characteristics of a surface element that is equally well captured by all cameras. This is an over-simplification that will be further discussed and refined in Section 3.
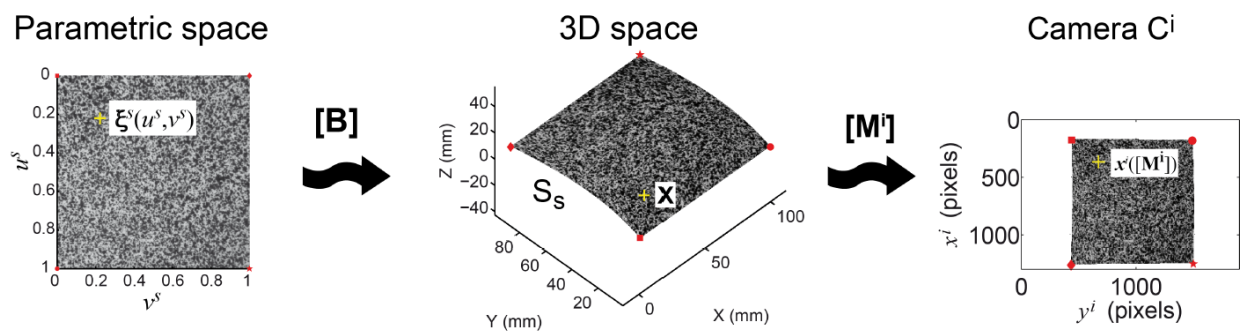

Figure 3: Determination of the projection matrices via global stereocorrelation for a given cameras $\mathrm{C}^{i}$ and surface $\mathrm{S}_{s}$. Here an intrinsic texture is used as the average of the surface texture transported onto the specimen frame $\boldsymbol{X}$ and eventually in the parametric space $\boldsymbol{\xi}^{s}$

The algorithm used in this second formulation is quite close to the previous one with alternate steps. First, the projection matrices $\left[\boldsymbol{M}^{i}\right]$ are corrected, and then, the intrinsic texture is refreshed with the new determination. These two steps are repeated until convergence. 


\subsection{Shape correction}

The first guess of the 3D shape (i.e., its nominal freeform) is projected onto the $2 \mathrm{D}$ space using the previously measured calibration matrices $\left[\boldsymbol{M}^{i}\right]$ thereby transporting the parametric space coordinate system $\boldsymbol{\xi}^{s}, \boldsymbol{x}^{i}\left(\left[\boldsymbol{M}^{i}\right], \boldsymbol{X}\left(\boldsymbol{\xi}^{s}\right)\right)$. The pictures can be interpolated to create sub-pictures in the parametric space. A global approach to stereocorrelation [2] is performed by moving the control points $\boldsymbol{P}_{k}$ of the NURBS patches. These motions induce pseudodisplacements visible for all cameras observing this surface, since the position in the specimen frame, $\boldsymbol{X}$ at fixed position in the parametric space $\boldsymbol{\xi}$, depends on the control points as detailed in Appendix A. To highlight this dependence, the control points are explicitly listed as arguments of the projection $\boldsymbol{x}^{i}\left(\left[\boldsymbol{M}^{i}\right], \boldsymbol{X}\left(\boldsymbol{P}_{k}, \boldsymbol{\xi}^{s}\right)\right)$. Let us however note that in this part the projection matrix $\left[\boldsymbol{M}^{i}\right]$ is known, and to simplify the notations, the latter dependence is omitted to write $\boldsymbol{x}^{i}\left(\boldsymbol{X}\left(\boldsymbol{P}_{k}, \boldsymbol{\xi}^{s}\right)\right)$. The apparent displacement in the $i$-th camera plane reads

$$
\delta \boldsymbol{x}^{i}\left(\boldsymbol{\xi}^{s}\right)=\frac{\partial \boldsymbol{x}^{i}}{\partial \boldsymbol{X}} \frac{\partial \boldsymbol{X}\left(\boldsymbol{P}_{k}, \boldsymbol{\xi}^{s}\right)}{\partial \boldsymbol{P}_{k}} \delta \boldsymbol{P}_{k}
$$

This equation defines new sensitivity fields in the same spirit as those introduced above. This leads to an estimate of the increment of control point positions $\delta \boldsymbol{P}_{k}$ that are added to the previous coordinates (Figure 4).

Paralleling the previous subsection, two formulations can be chosen. The first one exploits all possible image pairs for each surface. The second one makes use of a reference that is the average of the textures transported into the intrinsic parametric space. Because the latter formulation involves a more limited number of comparisons (i.e., $n_{c}$ rather than $n_{c}\left(n_{c}-1\right) / 2$ ), it 

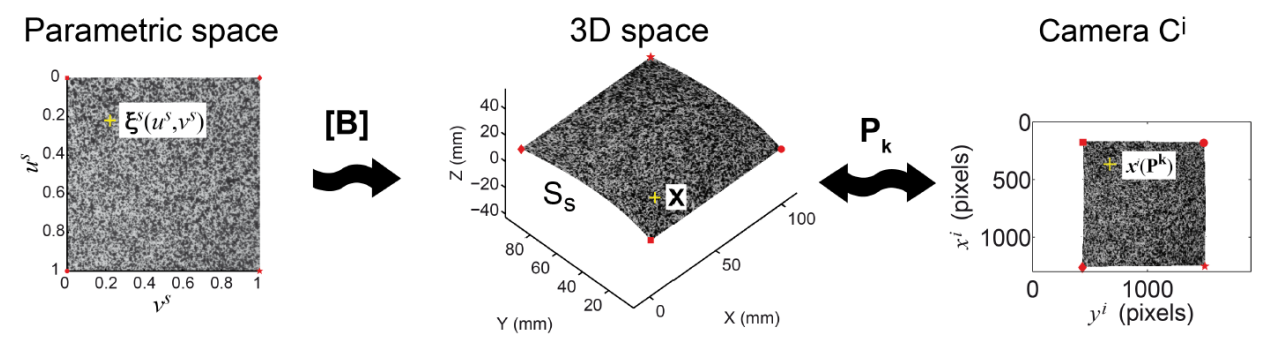

Figure 4: Measurement of the initial 3D shape via global stereocorrelation for a given camera $\mathrm{C}^{i}$ and surface $\mathrm{S}_{s}$ for which the intrinsic texture is updated in the parametric space $\boldsymbol{\xi}^{s}$

is the only one considered in the present study (although both have been implemented and tested).

The global stereocorrelation shape correction procedure consists of minimizing the sum of squared differences

$$
\left.\varrho^{2}\left(\delta \boldsymbol{P}_{k}\right)=\sum_{s=1}^{n_{s}} \sum_{i=1}^{n_{c}} \| f^{i}\left(\boldsymbol{x}^{i}\left(\boldsymbol{X}\left(\boldsymbol{P}_{k}, \boldsymbol{\xi}^{s}\right)\right)\right)-\hat{f}\left(\boldsymbol{\xi}^{s}\right)\right) \|^{2}
$$

with respect to each increment of control points gathered in $\left\{\delta \boldsymbol{P}_{k}\right\}$. The minimization is again achieved by resorting to Newton-Raphson's method alternating each iteration with an update of the intrinsic texture $\hat{f}$.

\subsection{D displacement field measurements}

In this section, the formulation of the 3D displacement field measurement via stereocorrelation is introduced. For the sake of simplicity, it is assumed hereafter that displacement fields are described in the same setting as the surface itself (i.e., the surface deformation is obtained by moving the control points). It is worth noting that other hypotheses can be made (e.g., finite 
element descriptions [13]). A global approach to stereocorrelation consists of minimizing the functional $\eta$

$$
\eta^{2}\left(\mathbf{d} \boldsymbol{P}_{\boldsymbol{k}}(t)\right)=\sum_{s=1}^{n_{s}} \sum_{i=1}^{n_{c}}\left\|g^{i}\left(\boldsymbol{x}^{i}\left(\boldsymbol{P}_{k}+\mathbf{d} \boldsymbol{P}_{\boldsymbol{k}}(t), \boldsymbol{\xi}^{s}\right)\right)-f^{i}\left(\boldsymbol{x}^{i}\left(\boldsymbol{P}_{k}, \boldsymbol{\xi}^{s}\right)\right)\right\|^{2}
$$

with respect to each coordinate motion $\mathbf{d} \boldsymbol{P}_{\boldsymbol{k}}(t)$ of the control points $\boldsymbol{P}_{k}$ for the $t$-th picture pair. In the present case, $f^{i}$ denotes the picture in the reference configuration for camera $\mathrm{C}^{i}$, and $g^{i}$ the picture from the same camera in the deformed configuration (Figure 5). These control point motions induce (true) displacements in the pictures (see Equation (6)).
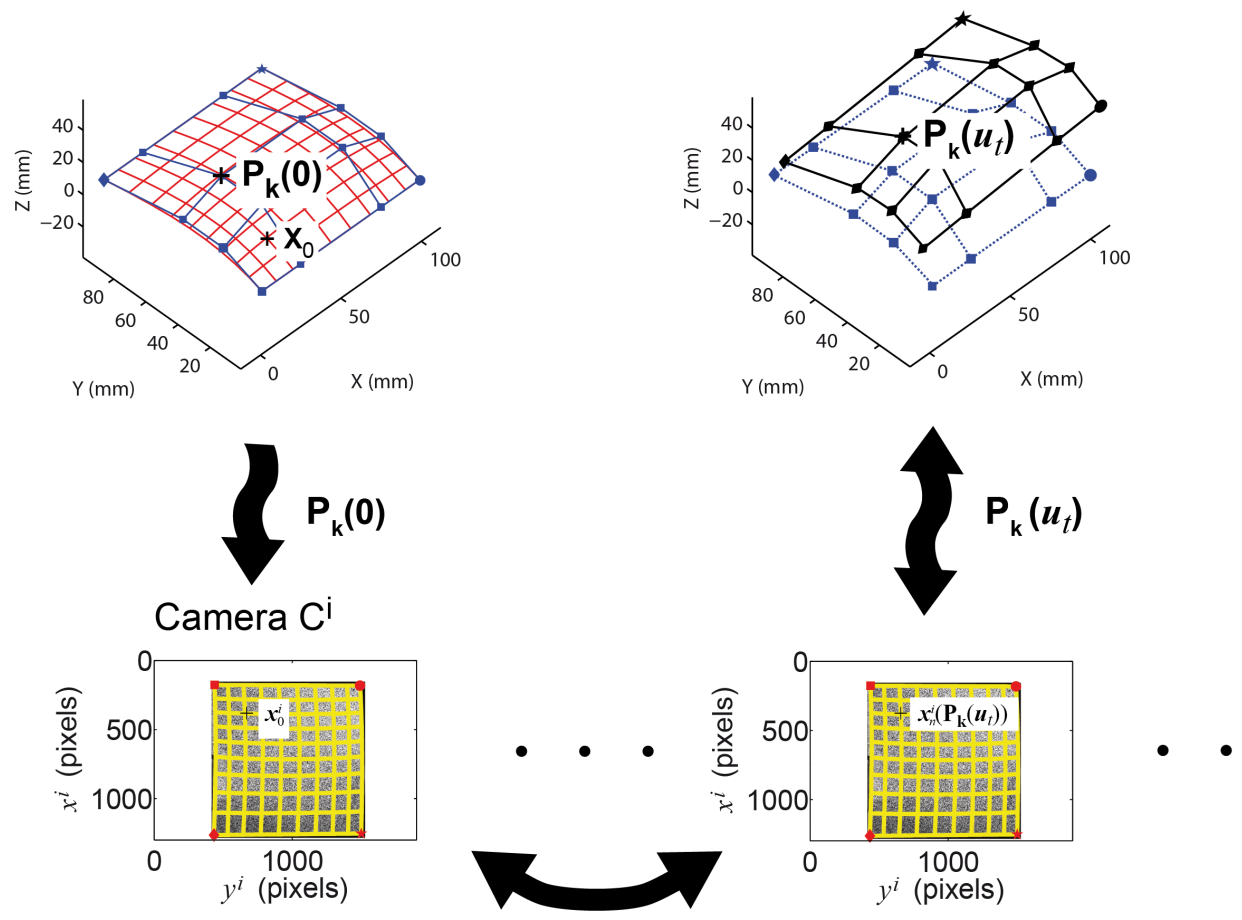

Figure 5: Measurement of 3D displacement fields via global stereocorrelation

Conversely, the true displacements are described within the language of CAD shape description of the surface. Although this may appear as restric- 
tive (there is no reason why the changes of shape should comply with the parameters chosen for designing its initial state), it is not. NURBSs are a powerful framework allowing for an easy addition or removal of control points, or local changes of the degree of regularity (e.g., continuous derivatives of chosen order) that can be tailored to fit an observed (i.e., continuous) displacement field at will. This enrichment can easily be pushed to the limit where preserving a good conditioning of the registration procedure can reveal difficult as for any other representation of the displacement field. Hence, the potential limitation is not a too stringent framework for describing the motion, but on the contrary the flexibility of the description leading to poor conditioning if too many degrees of freedom are included. However, in the present study, enrichment of the discretization is not considered, and it is assumed that the initial choice is wide enough to provide a fair description of the kinematics. The minimization is again performed by resorting to Newton-Raphson's scheme whose details will be provided in Section 3.

\section{Gray levels and pixels}

Up to this point, images were a theoretical abstraction where $f^{i}\left(\boldsymbol{x}^{i}\right)$ designates a scalar signature of the material point, $\boldsymbol{X}$, whose position in the $i$-th image plane is $\boldsymbol{x}^{i}$. For the sake of simplicity, $f^{i}$ was considered as being independent of the camera provided the position of the pixel would correctly be accounted for. The physics of image formation is however somewhat more complicated. Moreover, optimal performance of stereocorrelation requires the image texture to be "rough" (i.e., having very sharp gradients at the 
pixel level). This property is in conflict with what would be needed to neglect discretization (i.e., pixelization) effects. Thus, it is essential to express explicitly what is known or assumed with respect to image formation that could be camera-dependent. This procedure is the one that will allow for the formulation of "intrinsic" characteristics of the images that pertains to the observed material surface and not to the camera system, as well as features of the transformation that are camera-dependent. This partition is essential because when comparing images shot by different cameras only intrinsic features should be considered.

In the previous section, it was shown that each point in parametric space $\boldsymbol{\xi}^{s}$ was mapped onto a point in the specimen frame $\boldsymbol{X}\left(\boldsymbol{\xi}^{s}\right)$ via the blending functions (see Appendix A), and further mapped onto the image plane of camera $i, \boldsymbol{x}^{i}\left(\boldsymbol{\xi}^{s}\right)=\boldsymbol{x}^{i}\left(\boldsymbol{X}\left(\boldsymbol{\xi}^{s}\right)\right)$ with the projection matrices. These maps were used to transport the apparent texture $f^{i}\left(\boldsymbol{x}^{i}\right)$ onto the intrinsic one, $\hat{f}\left(\boldsymbol{\xi}^{s}\right)$. Therefore, it was assumed that $f^{i}\left(\boldsymbol{x}^{i}\left(\boldsymbol{\xi}^{s}\right)\right)$ could simply be related to $\hat{f}\left(\boldsymbol{\xi}^{s}\right)$ by a mere equality. This is at best an approximation, but it is important to go further in order to specify the details of how to design the norm $\|\cdots\|$ used previously in the $\varrho$ or $\eta$ functionals.

The actual $i$-th image is related to the intrinsic texture through a transformation $\mathcal{T}^{i}$ such that

$$
f^{i}\left(\boldsymbol{x}^{i}\left(\boldsymbol{\xi}^{s}\right)\right)=\mathcal{T}^{i s}\left[\hat{f}\left(\boldsymbol{\xi}^{s}\right)\right]
$$

where $\mathcal{T}^{i s}$ is generically nonlocal and nonlinear. Lens distortions are responsible for nonlocal corrections in the sense that pixel positions are not where one may naively assume they are. However, the importance of distortions has 
been discussed at length for stereovision [35] and can be measured beforehand so as to correct pictures as soon as they are captured. Hence distortions are assumed to be corrected in the sequel. Nonlocality also comes from the picture element, namely the pixel, that represents an elementary detector on the camera sensor, which integrates the intrinsic texture over some area. Optical effects such as out-of-focus regions give rise to blurring that is yet another form of nonlocality. Nonlocality could be ignored only if the gray level difference between consecutive pixels would be arbitrarily small, a property that is exactly the opposite of what is needed for high accuracy (i.e., high gray level difference between consecutive pixels ensures a small uncertainty). Nonlinearity comes from the response of the camera sensor, saturation effects, vignetting, non-ideally isotropic diffuse light scattering from the surface, or shadowing, not to mention hidden parts or obscuration.

A common ground for image registration would require $\mathcal{T}^{i s}$ to be invertible. Unfortunately, this is not the case. For instance, two points being mapped onto the very same pixel cannot be distinguished. However long wavelength features can be inverted, and hence those characteristics that can be shared (and hence compared) among images have to be precisely delineated.

\subsection{Pixel}

Pixels correspond to areas on the detector over which the light intensity is integrated. Hence, below the pixel scale the detailed information is lost. The map $\boldsymbol{x}^{i}\left(\boldsymbol{\xi}^{s}\right)$ may be used to transport the pixel size into the parametric space. 
The size of this pixel (or sizes as it is generically anisometric) and main axes orientation are to be known. In mathematical terms, the pixel can be seen as the ultimate differential element $\mathrm{d} \boldsymbol{x}^{i}$ that is mapped in the parametric space onto d $\boldsymbol{\xi}^{s}$ by the Jacobian

$$
\left[\mathbf{J}^{i}\right]=\frac{\mathrm{d} \boldsymbol{\xi}^{s}}{\mathrm{~d} \boldsymbol{x}^{i}}
$$

that conveys the information about the pixel size onto the parametric space (Figure 6).

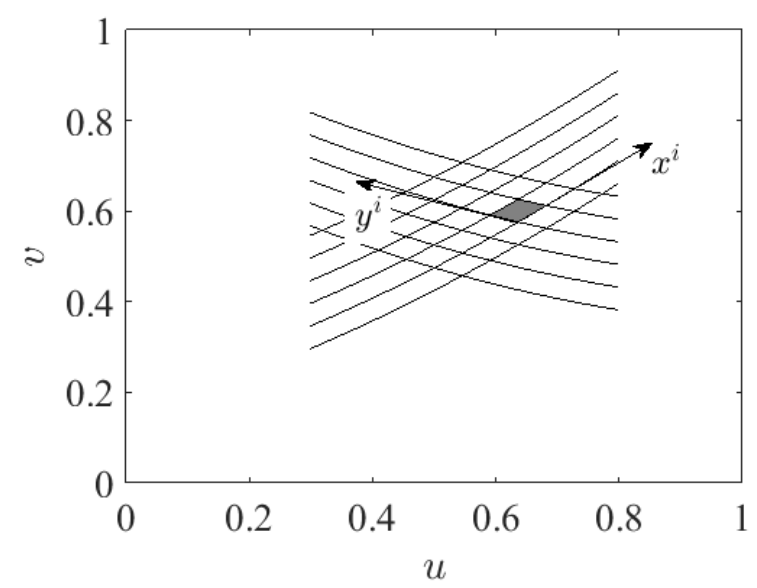

Figure 6: Mapping of a few lines and columns in camera $i$ detector array $\boldsymbol{x}^{i}=\left(x^{i}, y^{i}\right)$ shown in the intrinsic coordinate space $\boldsymbol{\xi}^{s}=(u, v)$. One pixel is shown as a gray shaded area. It constitutes a limit in the texture spatial resolution relative to one camera

The norms that were used in Equations (2), (4), (7) and (8) implied an integration over the $\boldsymbol{\xi}$ space. Because the texture is only known from the camera observation, there is no meaning to define a discretization in $\boldsymbol{\xi}$ space that would be finer than the mapping of the pixel size. The subtle point here is that different cameras will map their pixels onto a different $\mathrm{d} \boldsymbol{\xi}^{s}$. In 
the comparison between gray levels, a common spatial resolution, hence the coarser, is to be used.

In the example treated below, the above considerations become quite simple as the pixel resolution of all cameras observing a given surface will be quite comparable for symmetry reasons. However, the Jacobians will have two rather different eigenvalues, and hence the optimal point sampling in the parametric space will be anisotropic. Let us however stress that this is not a universal statement, and the above considerations lead naturally to an optimal intrinsic texture filtering and point sampling in $\boldsymbol{\xi}^{s}$ space.

\section{$3.2 \quad$ Gray levels}

Unfortunately, the discreteness of images is not the only aspect that makes the registration difficult. Another phenomenon comes from the fact that very often lighting is an issue, and one camera may receive a gray intensity that greatly differs from another camera image even if the pixel (and its surrounding) has correctly been described. Specular reflection of discrete light sources is only one aspect of the problem that may be partly accounted for or limited by using several light sources, optical diffusers, and matte surface finish.

A way to introduce a brightness modulation $\alpha\left(\boldsymbol{\xi}^{s}\right)$ in the norm $\|\cdots\|$, is to define for any field $\varphi\left(\boldsymbol{\xi}^{s}\right)$

$$
\|\varphi\|^{2}=\min _{\alpha\left(\boldsymbol{\xi}^{s}\right)} \iint\left[\varphi\left(\boldsymbol{\xi}^{s}\right)-\alpha\left(\boldsymbol{\xi}^{s}\right)\right]^{2} \mathrm{~d} \boldsymbol{\xi}^{s}
$$

where $\alpha$ is restricted to a low dimensional space of smooth function (e.g., polynomials of low order [22]). This norm gives less weight to very 
long range wavelengths where $\alpha$ can compensate $\varphi$ variations. In contrast, the high frequency content of $\varphi$, which generally controls the quality of image registration, is preserved. If a polynomial basis is chosen, then the minimization with respect to $\alpha\left(\boldsymbol{\xi}^{s}\right)$ is nothing but a least squares regression of $\varphi$, whose

classical residual L2-norm provides $\|\varphi\|^{2}$, in other terms, $\varphi\left(\boldsymbol{\xi}^{s}\right)-\alpha\left(\boldsymbol{\xi}^{s}\right)$ can be seen as a high-pass filtering of the texture.

Let us mention that contrast modulation may be treated the same way as a multiplicative field correcting the intrinsic texture, $\left(1+\beta\left(\boldsymbol{\xi}^{s}\right)\right) \varphi\left(\boldsymbol{\xi}^{s}\right)$, where the $\beta$ function is again a smooth function akin to $\alpha$. It is to be stressed that the above proposed measure of discrepancy is rather a seminorm than a norm, in the sense that some degrees of freedom, considered to be nonintrinsic to the surfaces are discarded from the comparison.

\subsection{Reference to intrinsic texture}

The above considerations also affect the way the second formulation of stereocorrelation is to be formulated. Rather than registering two images shot by two cameras, a procedure based on the registration between an image and the reference one in the parametric space was proposed. The reference image was initially introduced as the average of all available images of the same surface corrected from the projection as if the transformation $\mathcal{T}^{i s}$ were the identity. The present section is devoted to a discussion of what is intrinsic (and hence can be compared from one camera to another one) and what is camera-dependent (and should be discarded). Filtering at the common "pixel" size, and gray level transformations are to be accounted for before 
averaging.

However, one should beware of the fact that the exclusion of some features in the texture such as the mean gray level from the comparison, (or in mathematical terms the recourse to a semi-norm rather than a norm), may lead to an ill-posedness in the norm minimization if all unknowns are to be determined simultaneously. For instance, the mean gray level of the intrinsic texture is undefined. This difficulty is only apparent as the undetermined degrees of freedom, being extrinsic, have no influence on the registration. Various strategies can easily circumvent the problem, either by setting a convention to determine those floating unknowns, (e.g., the mean gray level of the intrinsic texture is set to 0 ) or by using a staggered procedure where different parts of the problem are treated sequentially. Along this second pathway, one may treat the $\alpha$-field as fixed in a first stage to perform the registration, and once registered, the $\alpha$-field is updated in a second stage. Repeating those two steps does not lead to degeneracy, albeit the mean intrinsic texture gray level ends up at an arbitrary value. This procedure is the one followed in the sequel.

\section{Proof of concept}

In order to demonstrate the feasibility of such a global stereocorrelation approach, an experiment is performed on a simple geometry using 4 DSLR cameras as sketched in Figure 1. Figure 7(a) shows the sample geometry composed of four orthogonal surfaces sharing their edges. The stereo-jig (see Figures 1 and $7(\mathrm{~b})$ ) is composed of four Canon EOS 60D cameras with 50- 
mm objective lenses. Each camera is observing one edge and two faces of the sample. This configuration allows us to obtain four stereo-jigs that are fully coupled via the CAD model of the observed surfaces. The geometry used herein is a multi-patch NURBS composed of 4 fourth order patches (i.e., the control net is made of $4 \times 4$ points). The whole geometry has $4 \times 3 \times 4 \times 3=144$ degrees of freedom.

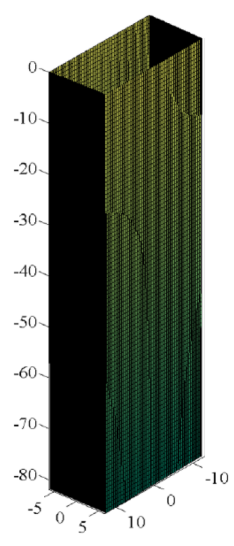

(a)

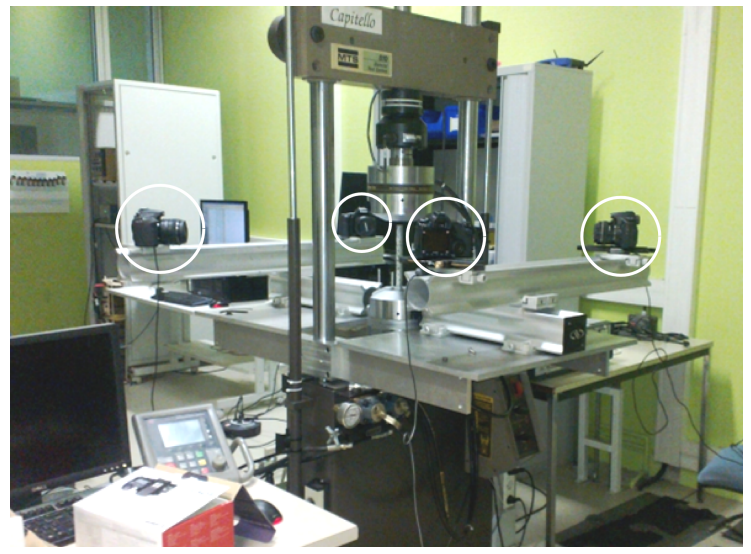

(b)

Figure 7: (a) Sample geometry. The coordinates are expressed in mm. (b) Experimental setup with 4 different DSLR cameras (circled in white) of a tensile test on an aluminum alloy bar

The second formulation is followed to perform the calibration of the full multi-view setup. Note however that in the present case, there is no major difference between the two formulations at convergence as the minimized functionals are proportional to each other when only 2 cameras can see each surface, under the same incidence and magnification

$$
\left\|f^{i}-\hat{f}\right\|^{2}=\left\|f^{i}-\frac{f^{i}+f^{j}}{2}\right\|^{2}=\frac{\left\|f^{i}-f^{j}\right\|^{2}}{4}
$$


This form allows brightness corrections to be accounted for very easily. The only difference that may be expected is prior to complete convergence, where each image is compared to a "compromise" between both versions of the intrinsic texture, and hence the basin of convergence is expected to be broader, leading to a more robust and faster convergence scheme. This point has however not been validated.

Figure 8(a) shows the correlation residuals on the sample geometry prior to the camera calibration procedure. It corresponds to the initialization step of the calibration procedure for which 6 remarkable features (i.e., two points on each edge seen by a camera) are manually selected by the user. From this selection, a first set of projection matrices is determined. The RMS correlation residual at the beginning of the computations was equal to $11 \%$ of the dynamic range of the cameras, and $3 \%$ at the end of the self-calibration step. The low value of residual is due to the fact that gray level corrections have been applied. In the present case, a bilinear field is chosen (i.e., a single NURBS patch of order 2) for each considered surface. The random texture is fully matched since it disappears in large areas of the reconstructed surface (Figure $8(\mathrm{~b})$ ). This result validates the proposed framework based upon the search for the intrinsic texture (i.e., second formulation).

Let us stress that three pairs of cameras would be sufficient to calibrate the geometrical model for the four cameras, i.e., positioning the coordinates of the camera focal point and optical axis. The use of the fourth pair introduces a redundant information, which is naturally accounted for in the proposed framework, to obtain the best determination of extrinsic parameters of the cameras (i.e., describing the transformation between the coordinate 


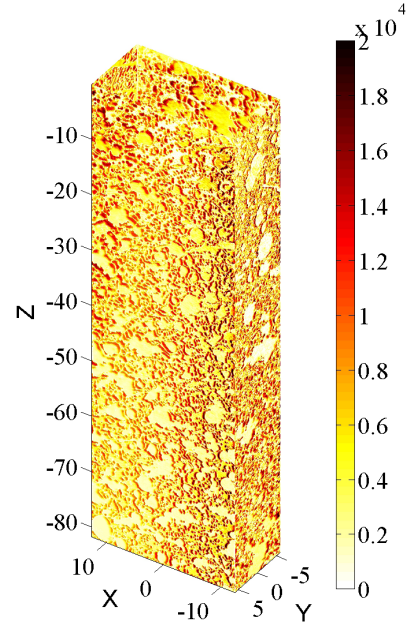

(a)

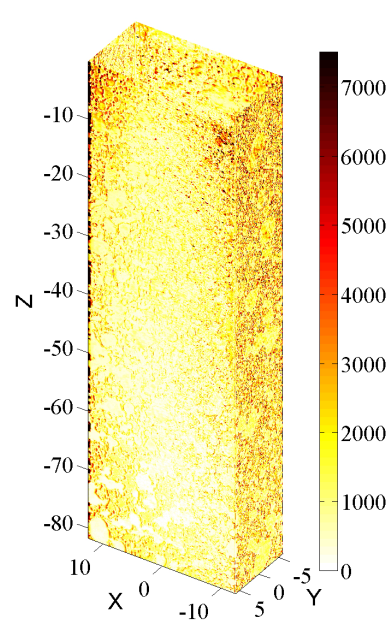

(b)

Figure 8: Correlation residuals (a) at the beginning of the calibration step ( $\eta$ $=11.16 \%)$ and $(\mathrm{b})$ at the end of the calibration step $(\eta=3.00 \%)$. The dynamic range of the registered pictures spans over 16-bits. Note that different dynamic ranges are used in the two subfigures

system of the NURBS model and that of each camera) without breaking the uniformity of their role (i.e., no "master" camera is chosen). Further weighing will offer the opportunity to account for the trustfulness of the projection depending on the direction of the surface normal with respect to the optical axis.

Differences between the measured and the nominal shape are shown in Figure 9. The differences are higher on the edges, because the sample shape does not fully match the geometrical model used. The RMS height differences between the nominal model and the reconstructed shape is equal to $8 \mu \mathrm{m}$, which is very low. The interested reader will find other validations of such 


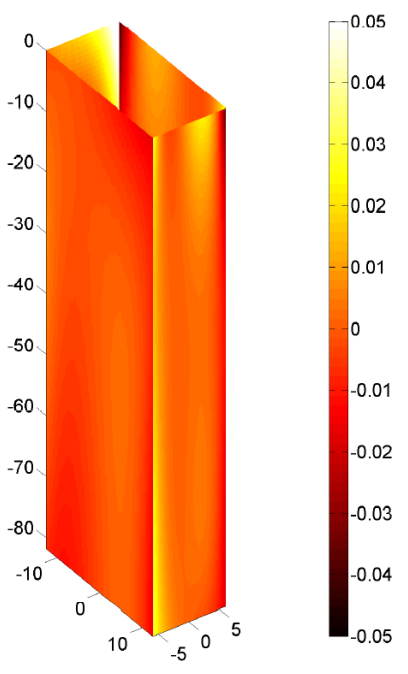

(a)

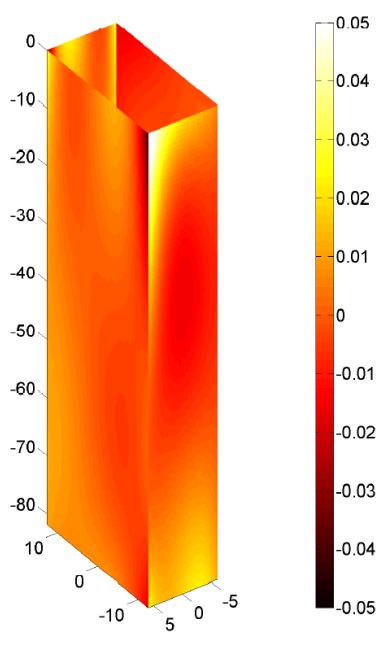

(b)

Figure 9: Differences (in $\mathrm{mm}$ ) along the normal to the surface between the reconstructed 3D shape and the nominal model. Differences are higher along the edges, where the model does not match the sample shape. Two views are shown when rotated $180^{\circ}$ about the longitudinal axis

an approach with only two cameras in Ref. [2].

The formulation (8) is followed to measure 3D displacement fields of the whole external surfaces of the sample. Figure 10 shows the displacement along the vertical axis. A small rotation can be noted in addition to the field associated with pure tension.

To validate these results, the residual maps $\tau$ are shown for the four cameras in Figure 11. The residuals are very low for all four cameras. The registration is deemed successful. The levels of the residuals are higher on the vertical edges of the surfaces, which is due to the difference between the real shape (slightly curved along the edges) and the geometric model used 


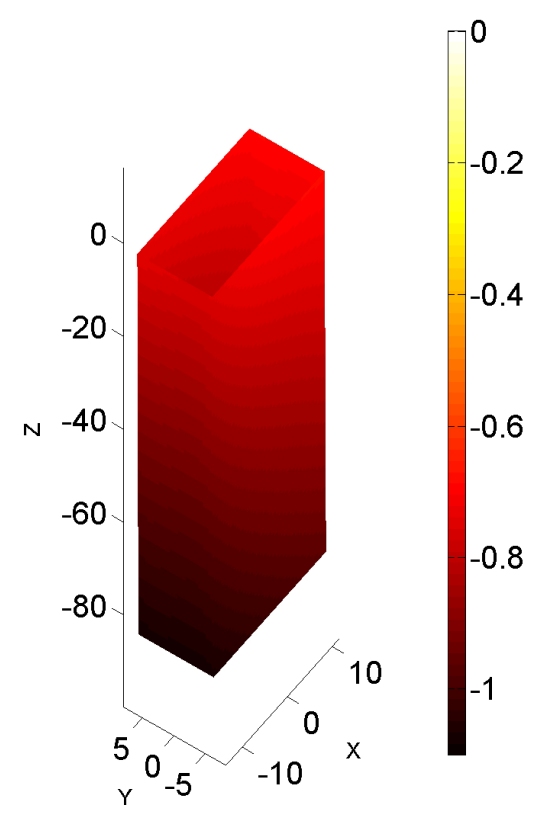

Figure 10: Longitudinal component of the displacement field (expressed in $\mathrm{mm}$ ) corresponding to the highest loading level of the tensile experiment

herein that does not account for that. New control points would have to be added to lower the residuals [12]. It was not performed herein. Other validations have been reported with only two cameras in Ref. [11].

\section{$5 \quad$ Isogeometric model updating (IGMU)}

In this part, an identification method is proposed by coupling CAD-based stereocorrelation and IGAs. The spirit is essentially the same as FEMU but applied to isogeometric analyses. It is therefore referred to as IsoGeometric Model Updating (or IGMU). CAD-based stereocorrelation mainly uses NURBS to describe 3D surfaces and 3D displacement fields. It is thus desir- 


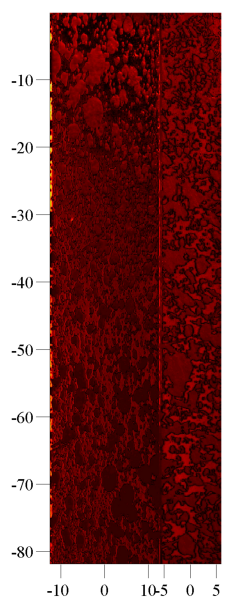

(a) camera 1

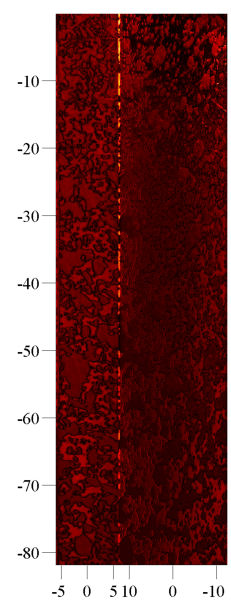

(b) camera 2

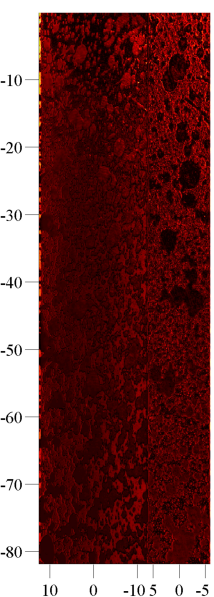

(c) camera 3

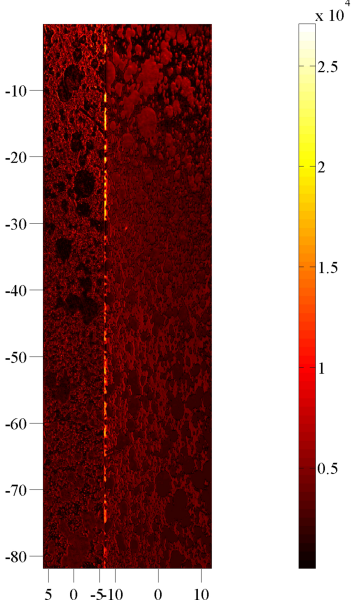

(d) camera 4

Figure 11: Gray level residuals $\tau$ for all four cameras corresponding to the highest loading level of the tensile experiment. The dynamic range of the registered pictures spans over 16-bits

able to have the same formulation for both experimental and numerical tools in order to make the comparison straightforward (i.e., avoiding interpolation, reprojection, or any unnecessary manipulation of the data). This is possible by resorting to an isogeometric code [10].

Figure 12 illustrates the principle of IGMU. The measured (Dirichlet) boundary conditions are prescribed to the IGA model. As in a FEMU procedure, displacement and load sensitivities with respect to the sought parameters are computed as finite differences to minimize the chi-squared residuals via, say, a Newton-Raphson scheme [18]. The material parameters minimizing the cost function are kept, and they can even be used to run a sensitivity analysis to acquisition noise [17].

In the present study, displacement fields $\boldsymbol{U}$ are parameterized by the po- 


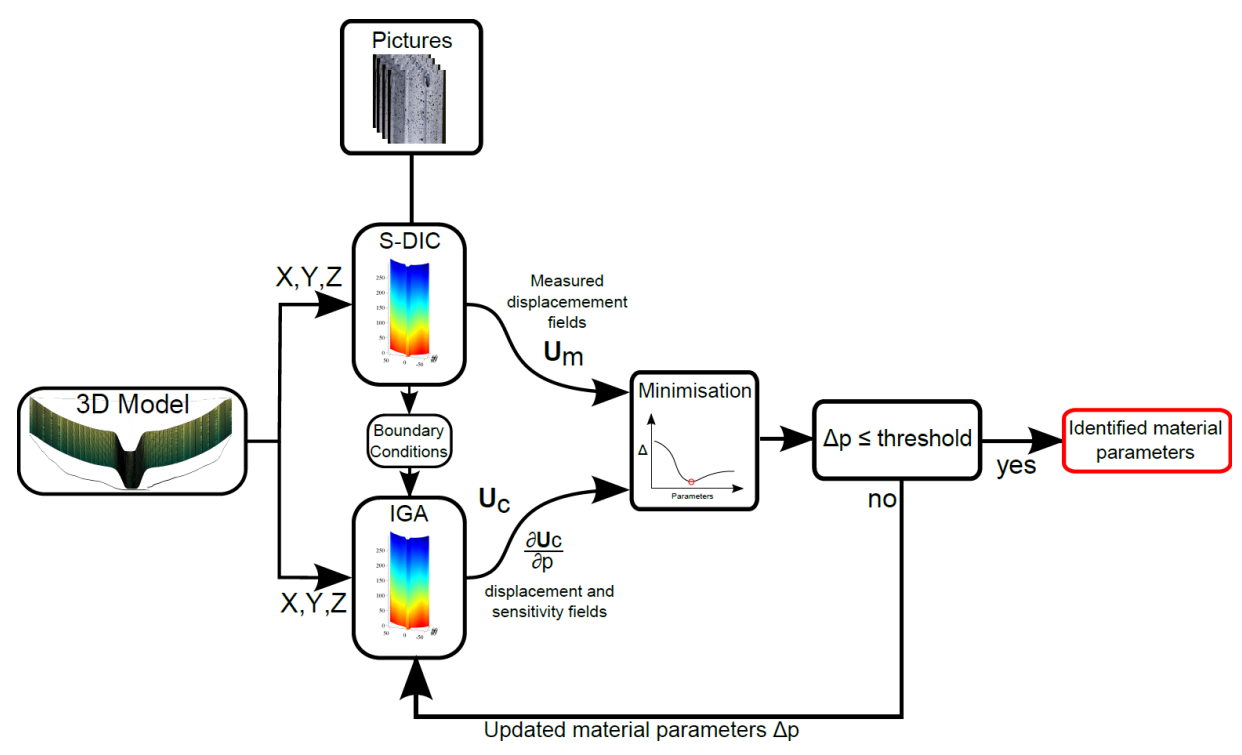

Figure 12: Principle of mechanical identification using IGMU

sition of the control points $\{\boldsymbol{P}\}$ here gathered as a vector for all surfaces and control points $k$. For simplicity, these parameters will be called "displacements," although it is clear that the actual displacement $\boldsymbol{U}$ is computed from the control points positions using the NURBS formalism. The measured displacements $\left\{\boldsymbol{P}^{m}\right\}$ are to be compared to the computed ones $\left\{\boldsymbol{P}^{c}\right\}$ by minimizing the following chi-squared error

$$
\chi_{p}^{2}(\{\boldsymbol{p}\})=\left(\left\{\boldsymbol{P}^{m}\right\}-\left\{\boldsymbol{P}^{c}\right\}\right)^{\dagger}[\boldsymbol{C}]\left(\left\{\boldsymbol{P}^{m}\right\}-\left\{\boldsymbol{P}^{c}\right\}\right)
$$

where $\{\boldsymbol{p}\}$ is the vector gathering all unknown material parameters used for the computation of $\left\{\boldsymbol{P}^{c}\right\}$. The chi-squared error is based on the $[\boldsymbol{C}]$ matrix resulting from the assembly of all elementary matrices according to the chosen formulation as described in Appendix B, Equation (27), as it can be shown that it is proportional to the inverse covariance matrix of the parameters $\{\boldsymbol{P}\}$ for a white noise on the images [21]. 
Finding the best set of parameters $\{\boldsymbol{p}\}$ consists of seeking the minimum of $\chi_{p}^{2}$. The computed displacement field at the $q$-th iteration is written as

$$
\boldsymbol{P}_{k}^{c}\left(\left\{\boldsymbol{p}^{(q)}\right\}, t\right)=\boldsymbol{P}_{k}^{c}\left(\left\{\boldsymbol{p}^{(q-1)}, t\right\}\right)+\frac{\partial \boldsymbol{P}_{k}^{c}}{\partial\{\boldsymbol{p}\}}\left(\left\{\boldsymbol{p}^{(q-1)}, t\right\}\right)\{\delta \boldsymbol{p}\}
$$

The sensitivity fields $[\boldsymbol{S}]=\frac{\partial\left\{\boldsymbol{P}^{c}\right\}}{\partial\{\boldsymbol{p}\}}$ are numerically computed by resorting to an IGA code [10] and forward finite differences. A new set of sensitivity fields is computed at each iteration of the algorithm. Using these fields, the corrections of the sought parameters read

$$
\{\delta \boldsymbol{p}\}=\left(\left[\boldsymbol{S}^{(q-1)}\right]^{\dagger}[\underline{\boldsymbol{C}}]\left[\boldsymbol{S}^{(q-1)}\right]\right)^{-1}\left[\boldsymbol{S}^{(q-1)}\right]^{\dagger}[\underline{\boldsymbol{C}}]\left(\left\{\boldsymbol{P}^{m}\right\}-\left\{\boldsymbol{P}^{c(q-1)}\right\}\right)
$$

where $\left[\boldsymbol{S}^{(q-1)}\right]$ gathers all sensitivity fields, $[\underline{\boldsymbol{C}}]$ gathers correlation matrices (used in the stereocorrelation measurement) for all time steps.

In practice, as only surface measurements are available via stereocorrelation, assumptions have to be make in order to prescribe Dirichlet boundary conditions to the computation. Depending on how much information is available (i.e., how many surfaces are analyzed) the method to create the surface of prescribed displacement used as a boundary condition is different. If two opposed surfaces are to be used, a simple linear interpolation between these two surfaces can be considered. If more surfaces are to be observed, the interpolation between the measured curves is performed using Coons interpolation and the surface of Dirichlet conditions is created using a Coons patch [8], see Figure 13. 


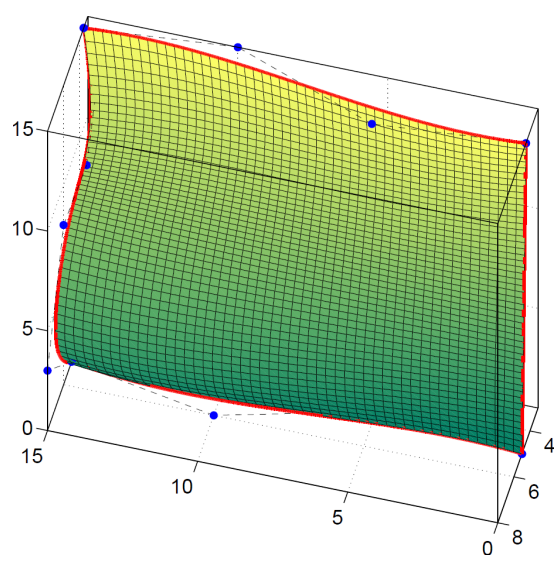

Figure 13: Illustration of the creation of a surface of prescribed displacements using four NURBS curves to define its boundaries thanks to a Coons patch

\subsection{Virtual experiment}

In order to validate the method, a virtual experiment has been created, consisting of a simulated tensile test on a virtual beam sample (see Figure 14(a)). To measure the displacement fields, pictures taken by two virtual stereo-jigs observing two opposed surfaces of the sample are determined using previously determined camera parameters (see Figure 14(b)). To generate synthetically deformed images, a reference one is created in the parametric space of each surface (Figure 3). In the present case, it corresponds to a true random texture of a biaxial experiment on a cross-shaped sample [30]. With the blending functions (see Appendix B), it is mapped onto the 3D shape, and projected onto the image plane with the projections matrices. From the computed projections, the gray levels are interpolated for each considered pixel location. The same approach is followed to compute the set of pictures for the deformed configurations. This method is easy to implement but induces 
more interpolation errors than other techniques such as ray-tracing [16]

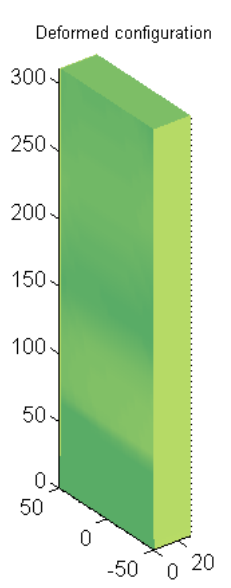

(a)

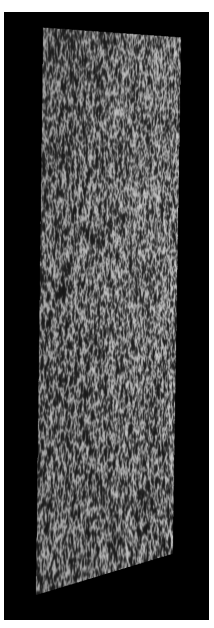

(b)

Figure 14: (a) Virtual beam model used in the experiment in its deformed state. (b) Example of an image shot by a virtual camera

The 3D displacement fields are measured on two opposite surfaces of the sample using the CAD-based stereocorrelation technique described earlier. Boundary conditions are extracted on the top and the bottom parts of each surface and then interpolated to create two surfaces that have prescribed displacements in an IGA code. The virtual experiment is then processed using the identification procedure described above. The two material parameters to be determined are the Young's modulus and Poisson's ratio. Figure 15 shows the change of these two quantities with the iteration number. Convergence is reached after a few iterations and the results are close to the prescribed values (i.e., $\approx 3 \%$ of the reference value).

Figure 16 shows the root mean square displacement error, i.e. difference between the known $\boldsymbol{U}_{c}$ and measured $\boldsymbol{U}_{m}$ displacement field at control points, 


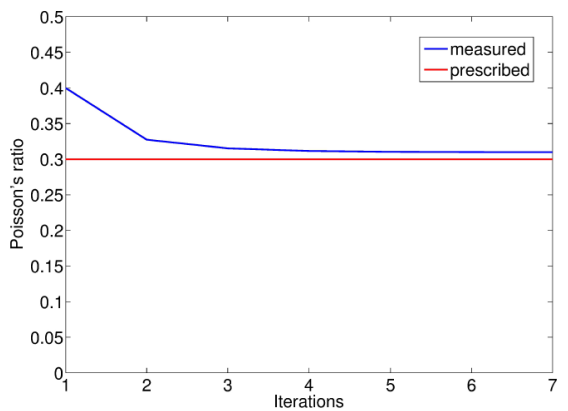

(a)

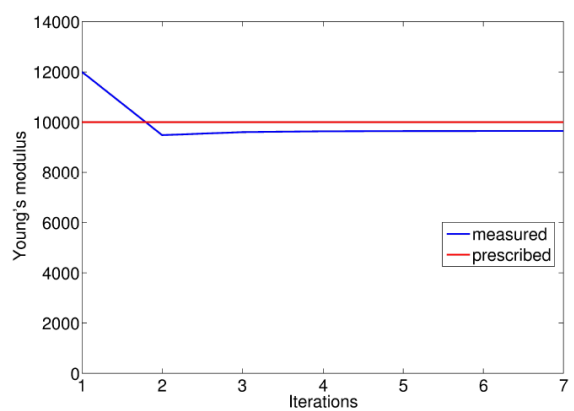

(b)

Figure 15: (a) Change of Poisson's ratio during the iterations (in blue) compared to the prescribed value (in red). (b) Change of Young's modulus during the iterations (in blue) compared to the prescribed value (in red)

as a function of the iterations. The level of error at convergence is very small (i.e., $47 \mu \mathrm{m}$ ), which validates the proposed framework. These errors may be due to inaccuracies and systematic bias in the image generation process.

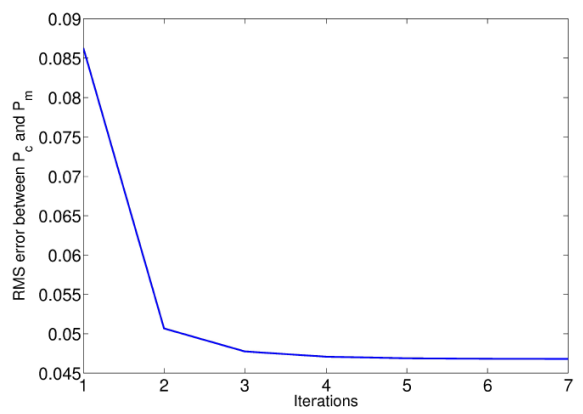

Figure 16: Change of the displacement error $\left\|\left\{\boldsymbol{P}_{m}\right\}-\left\{\boldsymbol{P}_{c}\right\}\right\|^{2}$ during the iterations 


\subsection{Analysis of the tensile test}

The tensile test is processed using the earlier described method. Two sets of initial parameters are chosen, which are given in Table 1, and then the identification procedure is run using the results obtained from the multiview stereocorrelation as measured displacement fields. Figure 18 shows the results in terms of Poisson's ratio and Young's modulus changes. It is observed that the levels at convergence are very close for the two different sets of parameters, although they are not those expected. The converged Young's modulus is higher than the expected value. The artificial rigidity introduced in the numerical model (by using too many Dirichlet boundaries in the IGA) can explain part of this effect. Further, the error in the evaluation of the reaction forces may also be caused by a biased estimation of the actual loading direction, which may induce rigid body rotations that can be observed in Figure 10.

Table 1: Initial parameters used in the IGMU procedure

\begin{tabular}{|l|c|c|}
\hline & Young's modulus (GPa) & Poisson's ratio \\
\hline Set 1 & 90 & 0.05 \\
Set 2 & 60 & 0.35 \\
\hline
\end{tabular}

The difference between the estimated Poisson's ratio and the expected level is close to $40 \%$. A rough estimate of the strain uncertainties indicates that the measurements in the $X-Y$ directions are (at least) 4 times that in the $Z$-direction, which is of the order of $150 \mu \epsilon$. Consequently, the transverse strain resolution is of the order of $600 \mu \epsilon$. The maximum longitudinal strain 


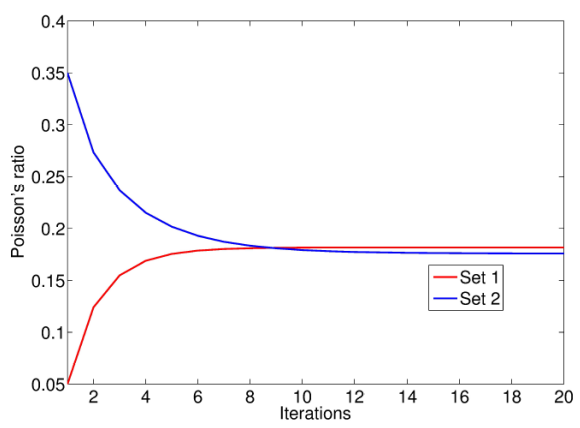

(a)

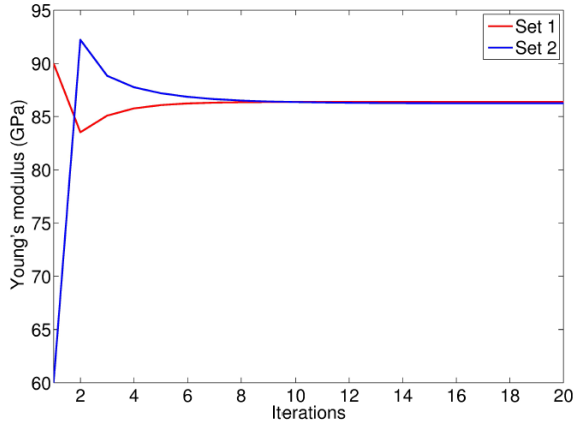

(b)

Figure 17: (a) Change of the Poisson's ratio during the iterations (final values: $\nu=0.182$ (red), $\nu=0.176$ (blue)). (b) Change of the Young's modulus during the iterations (final values: $\mathrm{E}=86.4 \mathrm{GPa}$ (red), $\mathrm{E}=86.3 \mathrm{GPa}$ (blue)) for two sets of initial parameters given in Table 1

is of the order of $1000 \mu \epsilon$ and the absolute transverse strain of the order of $300 \mu \epsilon$, which is lower than the corresponding strain resolution. The determination of the Poisson's ratio is very difficult, and the IGMU framework does not provides a good determination of this parameter.

Figure 18 shows the change of the root mean square difference between the measured and computed displacements. The level at convergence is very small (i.e., $26.5 \mu \mathrm{m}$ ) thereby indicating that it is likely to be very close to the standard displacement resolution. This result confirms that the transverse strains are very difficult to capture with the present approach. To improve the determination of the Poisson's ratio, another framework has to be considered by using even less degrees of freedom in the measurement stage to significantly lower the measurement uncertainties. Such a procedure will directly rely on the images to avoid intermediate steps. 


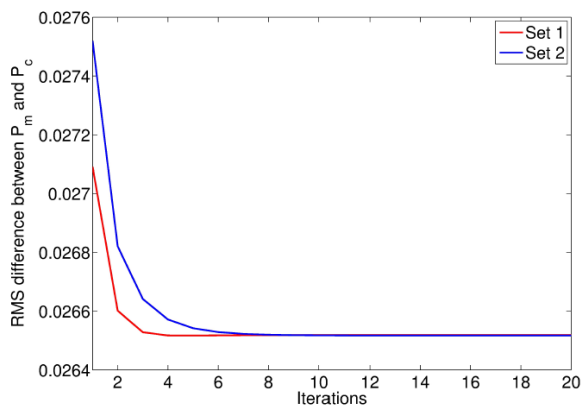

Figure 18: Change of the displacement error $\left\|\left\{\boldsymbol{P}_{m}\right\}-\left\{\boldsymbol{P}_{c}\right\}\right\|_{\mathbf{2}}^{2}$ during the iterations (final value: $26.5 \mu \mathrm{m}$ )

\section{$6 \quad$ Integrated approach (I-IGMU)}

A fully integrated approach is now developed. The kinematic basis then becomes the sensitivity fields to the sought material parameters [25, 31, 26]. The corresponding generalized degrees of freedom are the material parameters themselves, which are not as numerous as the degrees of freedom of the NURBS model of the four surfaces. Figure 19 shows the principle of integrated CAD-based stereocorrelation. The main difference with IGMU procedures is that the kinematic basis of the stereocorrelation procedure is now the set of sensitivity fields, parameterized by $\{\delta \boldsymbol{p}\}$, and no longer any arbitrary field parameterized by the control points $\{\delta \boldsymbol{P}\}$.

The registration problem of stereocorrelation consists of solving a series of linear systems

$$
\left[\boldsymbol{C}^{(i)}\right]\{\delta \boldsymbol{p}\}=\left\{\boldsymbol{d}^{(i)}\right\}
$$

with $\left[\boldsymbol{C}^{(i)}\right]=\left[\boldsymbol{S}^{(i)}\right]^{\dagger}[\underline{\boldsymbol{C}}]\left[\boldsymbol{S}^{(i)}\right]$, which contains the projection of the correlation matrix onto the sensitivity fields, and $\left\{\boldsymbol{d}^{(i)}\right\}=\left[\boldsymbol{S}^{(i)}\right]^{\dagger}\left\{\underline{\boldsymbol{d}}^{(i)}\right\}$ the projection 


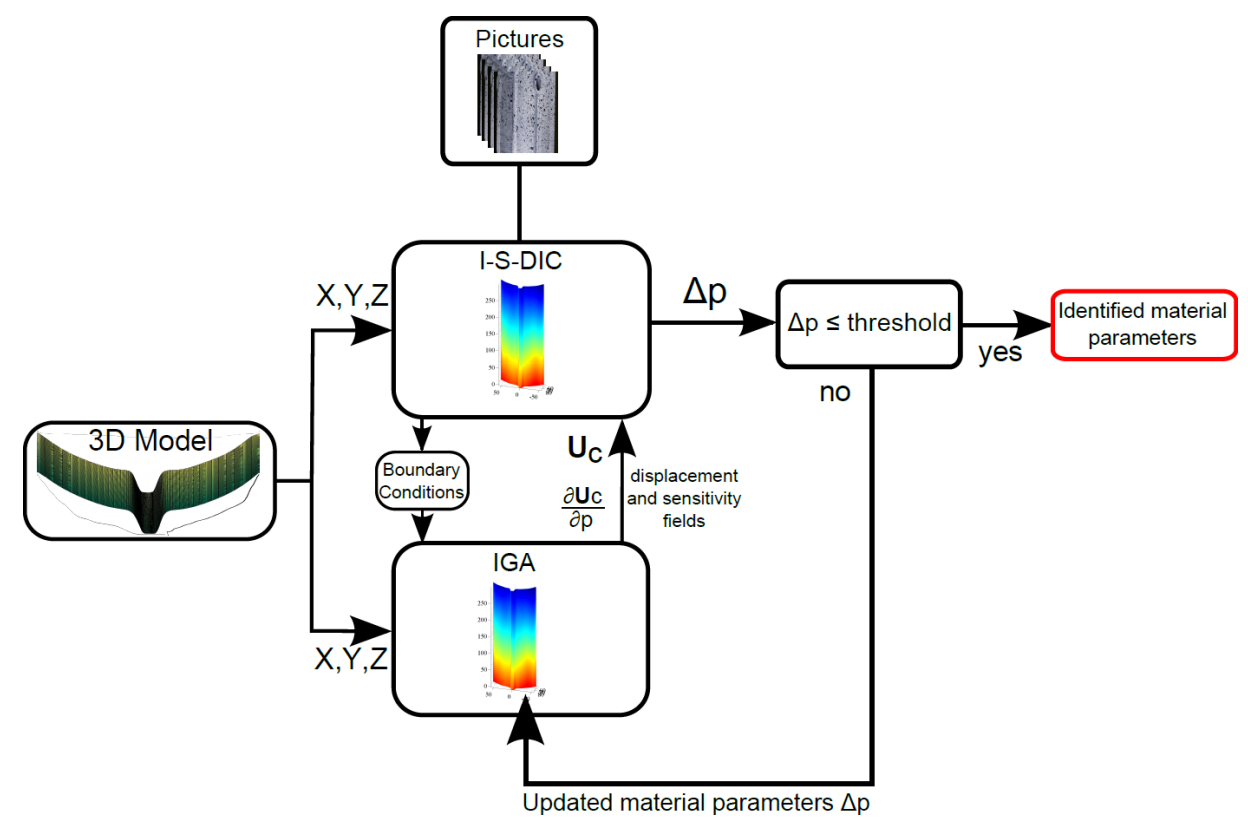

Figure 19: Identification using integrated CAD-based stereocorrelation

of the residual vector. It has to be noted that Equation (16) uses the same setting as standard DIC formulations, but the problem is directly expressed in terms of the material parameters rather than the kinematic degrees of freedom used previously in the CAD-based stereocorrelation.

\subsection{Virtual experiment}

The virtual experiment presented in Section 5.1 is analyzed again using the integrated approach and Figure 20 shows the change of the Poisson's ratio during the iterations of the integrated code. It can be noted that the use of integrated stereocorrelation significantly improves the quality of the identification as the final value is very close to the prescribed Poisson's ratio (i.e., $0.1 \%$ of error at convergence). 


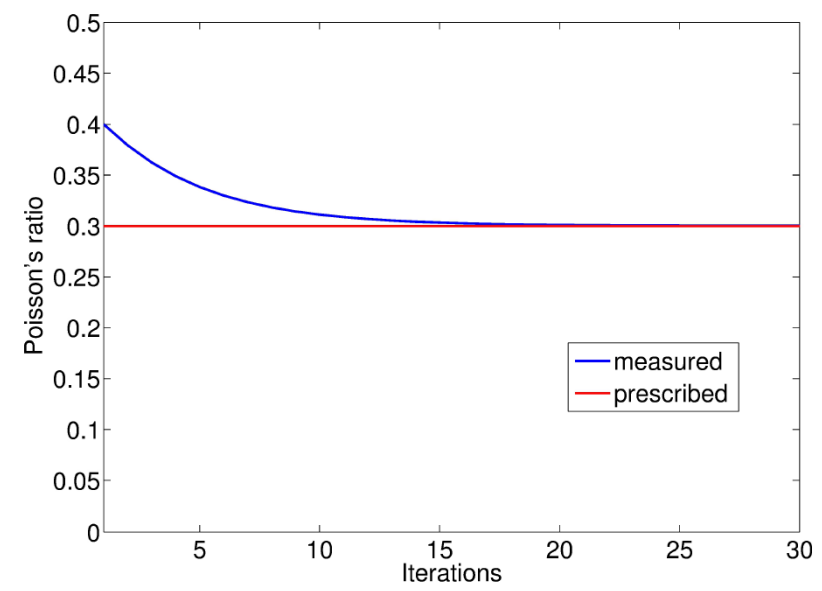

Figure 20: Change of Poisson's ratio during the iterations (in blue) compared to the prescribed value (in red). When convergence is reached, the level of error is close to $0.1 \%$ of the reference value, which is significantly lower than with the IGMU approach

\subsection{Analysis of the tensile test}

To validate the usefulness of the integrated approach on the extraction of Poisson's ratio from the tensile test, the change of the gray level residual divided by the dynamic range of the cameras is investigated and the results are shown in Figure 21. It is observed that a minimum is found around 0.29 for the Poisson's ratio, which is very close to the expected value. It should be noted that the values of the residual error does not change much for a large range of Poisson's ratio values (i.e., 0.27-0.31), which indicates that this case is extremely difficult even for an integrated approach. This result also explains that minute uncertainties may hinder the identification procedure as exemplified in the IGMU analysis. 


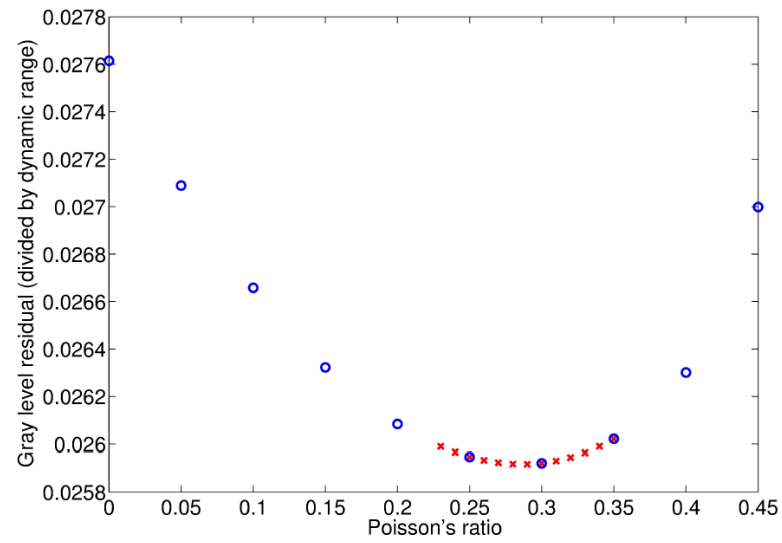

Figure 21: Change of the gray level error with respect to the Poisson's ratio. In blue, the range lies between 0 and 0.45 with increments of 0.05 . In red, the range is $0.23-0.35$ and the increment is 0.01 . The minimum is reached for $\nu=0.29$

In the present case, the value of the Young's modulus is identical to the previous one since the Dirichlet boundary conditions are the same in both approaches. It is worth noting that these boundary conditions can become additional unknowns in an integrated approach. This route was not followed herein.

\section{Summary and Outlook}

In this paper, a fully integrated framework combining stereovision, NURBSbased surface descriptions, isogeometric analyses to reconstruct dense 3D shapes, measure dense 3D displacement fields and identify material parameters has been presented. This unique combination is made possible thanks to 
various global stereo-DIC procedures in which the output is either expressed in terms of projection matrices during the calibration step, control points of a NURBS model for 3D shape and displacement measurements, or directly material parameters. This procedure constitutes a novel homogeneous framework bridging the gaps between CAD design and its validation or correction, mechanical tests and their modeling through isogeometric analyses.

Moreover, such a new framework is flexible enough to incorporate an arbitrary number of cameras, which is very beneficial to the analysis of real structures, as it allows for a complete observation of the specimen or structure. A new formulation has been introduced in which each camera is treated independently and a unique pattern expressed in the parametric space of the CAD model of each surface is transported from the registration of all available pictures. Having access to this additional piece of information makes the present procedure potentially more robust since it is much less prone to acquisition noise.

Let us also note that the CAD-model of the analyzed surfaces being the reference, all the reported results are shown in its unique frame. Consequently, no additional post-processing is needed to "stich" all stereocorrelation analyses (e.g., by resorting to procedures such as iterative closest point algorithms $[3,6,36,19])$. The latter ones are performed globally and the calibration step allowed all the camera frames to be related to that of the object of interest.

The proof of concept developed herein consists of the analysis of a tensile test on a rectangular bar both for a virtual experiment and for an actual tensile test. In both cases, identification procedures are developed either as a 
subsequent step after the measurement step by updating isogeometric analyses or in an integrated way by combining the measurement and identification steps. Both approaches are first validated by resorting to a virtual experiment. For the Young's modulus, higher than expected values are found. At least two reasons may explain such differences. First, the way the reaction forces are evaluated is delicate with the IGA code used herein. Second, the actual load direction is not necessarily aligned along the longitudinal direction of the sample. For the Poisson's ratio, the IGMU approach does not yield satisfactory levels because the transverse strain levels are very small. Conversely, the integrated approach by significantly reducing the number of degrees of freedom of the correlation procedure allows the Poisson's ratio to be identified in a very satisfactory way.

The results obtained herein need to be confirmed by the analysis of other experiments and more complex constitutive models. In particular, the measurement and identification resolutions need to be assessed to know whether the sought parameters are identifiable. The careful analysis of the sensitivity fields is needed as proposed in 2D-DIC frameworks $[17,26]$.

As a closure, let us note that from the design of an experiment to the validation of a new structure or assembly, all of the tools can be integrated into a unique toolbox for the modern experimentalist who more often than ever also utilizes CAD and FE softwares. This avoids creating "Babel towers" as seamless procedures can be developed without having to resort to cumbersome reprojections between experimentally extracted data and numerically computed fields. The framework developed herein aims to unify the worlds of experiments and simulations so that the emergence of "simulation-based 
engineering sciences" will be facilitated [27].

\section{Acknowledgements}

The authors would like to acknowledge SNECMA for supporting this research and Dr. Julien Schneider for stimulating discussions. This work is part of the "PRC Composite" research project supported by DGAC, and involving SAFRAN group, ONERA, and CNRS. 


\section{References}

[1] S. Avril, M. Bonnet, A.S. Bretelle, M. Grédiac, F. Hild, P. Ienny, F. Latourte, D. Lemosse, S. Pagano, E. Pagnacco, and F. Pierron. Overview of identification methods of mechanical parameters based on full-field measurements. Exp. Mech., 48(4):381-402, 2008.

[2] B. Beaubier, J.E. Dufour, F. Hild, S. Roux, S. Lavernhe-Taillard, and K. Lavernhe-Taillard. CAD-based calibration of a 3D-DIC system: Principle and application on test and industrial parts. Exp. Mech., 54(3):329$341,2014$.

[3] P.J. Besl and N.D. McKay. A method for registration of 3-D shapes. IEEE Trans. Patt. Anal. Mach. Intell., 14(2):239-256, 1992.

[4] G. Besnard, F. Hild, and S. Roux. "Finite-element" displacement fields analysis from digital images: Application to Portevin-Le Châtelier bands. Exp. Mech., 46:789-803, 2006.

[5] G.B. Broggiato. Adaptive image correlation technique for full-field strain measurement. In C. Pappalettere, editor, 12th Int. Conf. Exp. Mech., pages 420-421. McGraw Hill, Lilan (Italy), 2004.

[6] Y. Chen and G. Medioni. Object modelling by registration of multiple range images. Image Vis. Comput., 10(3):145-155, 1992.

[7] J.D. Collins, G.C. Hart, T.K. Hasselman, and B. Kennedy. Statistical identification of structures. AIAA J., 12(2):185-190, 1974. 
[8] S. Coons. Surface patches and B-spline curves. In R.E. Barnhill and R.F. Riesenfeld, editors, Computer Aided Geometric Design, pages 1-16. Academic Press, 1974.

[9] J.A. Cottrell, T.J.R. Hughes, and Y. Bazilevs. Isogeometric Analysis: Toward Integration of CAD and FEA. Wiley, 2009.

[10] C. de Falco, A. Reali, and R. Vázquez. GeoPDEs: A research tool for isogeometric analysis of PDEs. Adv. Eng. Software, 42(12):1020-1034, 2011.

[11] J.-E. Dufour, B. Beaubier, F. Hild, and S. Roux. CAD-based measurement of displacement and strain fields. Principle and first validation. Exp. Mech., submitted for publication, 2015.

[12] J.-E. Dufour, B. Beaubier, F. Hild, S. Roux, and S. Leclercq. Shape measurement using CAD-based stereo-DIC. In H. Jin, C. Sciammarella, S. Yoshida, and L. Lamberti, editors, Advancement of Optical Methods in Experimental Mechanics, Volume 3, Conference Proceedings of the Society for Experimental Mechanics Series, pages 333-338. Springer, 2014.

[13] J.E. Dufour, B. Beaubier, S. Roux, and F. Hild. Displacement measurement using CAD-based stereo-correlation with meshes. In ICEM conference, 2014.

[14] O. Faugeras. Three-dimensional computer vision: a geometric viewpoint. MIT Press, Cambridge, MA (USA), 1993. 
[15] O.D. Faugeras, Q.T. Luong, and S.J. Maybank. Camera self-calibration: Theory and experiments. In Proc. 2nd ECCV, pages 321-334. SpringerVerlag, 1992.

[16] D. Garcia, J.J. Orteu, L. Robert, B. Wattrisse, and F. Bugarin. A generic synthetic image generator package for the evaluation of $3 \mathrm{~d}$ digital image correlation and other computer vision-based measurement techniques. In Proc. Photomechanics 2013. http://hal.archivesouvertes.fr/hal-00836324, 2013.

[17] R. Gras, H. Leclerc, F. Hild, S. Roux, and J. Schneider. Identification of a set of macroscopic elastic parameters in a 3D woven composite: Uncertainty analysis and regularization. Int. J. Solids Struct., 55:2-16, 2015.

[18] M. Grédiac and F. Hild, editors. Full-Field Measurements and Identification in Solid Mechanics. ISTE / Wiley, London (UK), 2012.

[19] J. Harvent, B. Coudrin, L. Brèthes, J.-J. Orteu, and M. Devy. Multiview dense 3D modelling of untextured objects from a moving projectorcameras system. Mach. Vis. Appl., 24(8):1645-1659, 2013.

[20] F. Hild and S. Roux. Digital image correlation: From measurement to identification of elastic properties - A review. Strain, 42:69-80, 2006.

[21] F. Hild and S. Roux. Comparison of local and global approaches to digital image correlation. Exp. Mech., 52(9):1503-1519, 2012. 
[22] F. Hild and S. Roux. Digital Image Correlation, pages 183-228. WileyVCH, Weinheim (Germany), 2012.

[23] K.T. Kavanagh. Extension of classical experimental techniques for characterizing composite-material behavior. Exp. Mech., 12(1):50-56, 1972.

[24] K.T. Kavanagh and R.W. Clough. Finite element applications in the characterization of elastic solids. Int. J. Solids Struct., 7:11-23, 1971.

[25] H. Leclerc, J.N. Périé, S. Roux, and F. Hild. Integrated digital image correlation for the identification of mechanical properties, volume LNCS 5496, pages 161-171. Springer, Berlin (Germany), 2009.

[26] F. Mathieu, H. Leclerc, F. Hild, and S. Roux. Estimation of elastoplastic parameters via weighted FEMU and integrated-DIC. Exp. Mech., 55(1):105-119, 2015.

[27] Blue Ribbon Panel. Simulation-based engineering sciences. Final report, NFS (www.nsf.gov/pubs/reports/sbes_final_report.pdf), 2006.

[28] L. Piegl and W. Tiller. The NURBS Book - 2nd Edition. Springer, 1997.

[29] S. Roux and F. Hild. Stress intensity factor measurements from digital image correlation: Post-processing and integrated approaches. Int. J. Fract., 140(1-4):141-157, 2006.

[30] S. Roux and F. Hild. Digital image mechanical identification (dimi). Exp. Mech., 48(4):495-508, 2008. 
[31] J. Réthoré. A fully integrated noise robust strategy for the identification of constitutive laws from digital images. Int. J. Num. Meth. Eng., 84(6):631-660, 2010.

[32] Y. Sun, J. Pang, C. Wong, and F. Su. Finite-element formulation for a digital image correlation method. Appl. Optics, 44(34):7357-7363, 2005.

[33] M.A. Sutton. Computer vision-based, noncontacting deformation measurements in mechanics: A generational transformation. Appl. Mech. Rev., 65(AMR-13-1009, 050802), 2013.

[34] M.A. Sutton and F. Hild. Recent advances and perspectives in digital image correlation. Exp. Mech., 55(1):1-8, 2015.

[35] M.A. Sutton, J.J. Orteu, and H. Schreier. Image correlation for shape, motion and deformation measurements: Basic Concepts, Theory and Applications. Springer, New York, NY (USA), 2009.

[36] Z. Zhang. Iterative point matching for registration of free-form curves and surfaces. Int. J. Comput. Vis., 13(2):119-152, 1994. 


\section{Appendix A: NURBS framework}

A NURBS patch is defined by its order, a network of control points with associated weights, and its knot vector. The surface $\boldsymbol{X}(u, v)=(X, Y, Z)$ is expressed in the parametric space $(u, v)$ as

$$
\boldsymbol{X}(u, v)=\sum_{i=0}^{m} \sum_{j=0}^{n} B_{i j}(u, v) \boldsymbol{P}_{\boldsymbol{i} \boldsymbol{j}}
$$

where the blending functions are expressed as

$$
B_{i j}(u, v)=\frac{N_{i, p}(u) N_{j, q}(v) \omega_{i j}}{\sum_{i=0}^{m} \sum_{j=0}^{n} N_{i, p}(u) N_{j, q}(v) \omega_{i j}}
$$

with

$$
\forall u \in[0,1], N_{i, 0}(u)=\left\{\begin{array}{l}
1 \text { when } u_{i} \leq u \leq u_{i+1} \\
0 \text { otherwise }
\end{array}\right.
$$

and

$$
N_{i, p}(u)=\frac{u-u_{i}}{u_{i+p}-u_{i}} N_{i, p-1}(u)+\frac{u_{i+p+1}-u}{u_{i+p+1}-u_{i+1}} N_{i+1, p-1}(u),
$$

where $N_{i, p}$ are mixing functions, $\boldsymbol{P}_{i j}$ the coordinates of control points of the surface, $\omega_{i j}$ corresponding weights, $(m+1) \times(n+1)$ the number of control points and $(p, q)$ the degrees of the surface. 


\section{Appendix B: Norm minimization}

In Section 2, the projection matrices $[\boldsymbol{M}], 3 \mathrm{D}$ shape or 3D displacements defined through the motion of control points $\boldsymbol{P}_{k}$ were optimized from the minimization of the norm of image differences. It is worth emphasizing that these problems are strongly nonlinear as the variables to be optimized affect the argument of fields describing the texture of the observed sample, texture that is usually a very irregular pattern. However, because the parameterization is well suited to the problem, a Newton-Raphson scheme is used, and this section aims to provide explicit expressions of the iterative linearized problem to solve at each iteration of the this minimization procedure.

In all three recourses to registration, for geometry assessment, for shape correction and for motion estimation, from equations such as such as Eq. (2), the quantity to be minimized for each surface and camera pair, $i$ and $j$, is the norm of a residual written as

$$
\theta^{i j}\left(\boldsymbol{\xi}^{s}\right)=f^{i}\left(\boldsymbol{x}^{i}\left(\boldsymbol{\xi}^{s}\right)\right)-f^{j}\left(\boldsymbol{x}^{j}\left(\boldsymbol{\xi}^{s}\right)\right)
$$

When a reference to an intrinsic image texture is used, such as mentioned for Equations (4), (7) and (8), the residual for each camera is the norm of the residual

$$
\tau^{i}\left(\boldsymbol{\xi}^{s}\right)=f^{i}\left(\boldsymbol{x}^{i}\left(\boldsymbol{\xi}^{s}\right)\right)-\hat{f}\left(\boldsymbol{\xi}^{s}\right)
$$

The previous subsections detailed how this norm is to be interpreted, that is (i) with a suited common spatial resolution, and (ii) tolerance to gray level variations corresponding to treating as additional degrees of freedom smooth fields, $\alpha\left(\boldsymbol{\xi}^{s}\right)$, so that finally, the norm of the above residual reduces to the 
minimum of the standard L2-norm of the modified residual, $\bar{\theta}^{i j}$

$$
\bar{\theta}^{i j}\left(\boldsymbol{\xi}^{s}\right)=f^{i}\left(\boldsymbol{x}^{i}\left(\boldsymbol{\xi}^{s}\right)\right)-f^{j}\left(\boldsymbol{x}^{j}\left(\boldsymbol{\xi}^{s}\right)\right)+\alpha^{i j}\left(\boldsymbol{\xi}^{s}\right)
$$

or $\bar{\tau}^{i}$

$$
\bar{\tau}^{i}\left(\boldsymbol{\xi}^{s}\right)=f^{i}\left(\boldsymbol{x}^{i}\left(\boldsymbol{\xi}^{s}\right)\right)-\hat{f}\left(\boldsymbol{\xi}^{s}\right)+\alpha^{i}\left(\boldsymbol{\xi}^{s}\right)
$$

Last, this norm is obtained numerically by an integration over $\boldsymbol{\xi}^{s}$, which has to be reduced to a discrete sum of the squared residual over sampling points $\boldsymbol{\xi}_{n}^{s}$, whose distance is ideally equal to the local effective common "pixel" size.

In addition to the $\alpha$ minimization, the use of this norm was to optimize the mapping $\boldsymbol{x}^{i}\left(\boldsymbol{\xi}^{s}\right)$ with respect to variations of either projection matrices or control point positions. In all cases, the variation of these parameters, here called $a_{k}$ and gathered in the column vector $\{\boldsymbol{a}\}$ (be they matrix elements $\{\boldsymbol{M}\}$ or component of control point displacement $\{\boldsymbol{P}\})$, induces a change of the mapping that can be seen as a velocity or sensitivity field in $\boldsymbol{\xi}^{s}$ plane

$$
\boldsymbol{S}_{k}^{i}\left(\boldsymbol{\xi}^{s}\right)=\frac{\partial \boldsymbol{x}^{i}\left(\boldsymbol{\xi}^{s}\right)}{\partial a_{k}}
$$

whose expression was detailed earlier (see Equations (3) and (6)).

The minimization of the above norm evaluated over surfaces $\mathrm{S}_{s}$ and camera pairs $\mathrm{C}^{i}$ and $\mathrm{C}^{j}$ (first formulation) consists of assembling different linear systems to obtain the corrections $\{\delta \boldsymbol{a}\}$ to their current estimate

$$
\left[\boldsymbol{C}^{s i j}\right]\{\delta \boldsymbol{a}\}=\left\{\boldsymbol{d}^{s i j}\right\}
$$

whereas for the second formulation the sum runs over surfaces $S_{s}$ and cameras $\mathrm{C}^{i}$

$$
\left[\boldsymbol{C}^{s i}\right]\{\delta \boldsymbol{a}\}=\left\{\boldsymbol{d}^{s i}\right\}
$$


The expressions of matrices $[\boldsymbol{C}]$ and second member $\{\boldsymbol{d}\}$ are now detailed for both formulations.

The second formulation is considered first as the notations are more compact. Let us introduce $\Gamma$ such that

$$
\Gamma_{k}^{i}\left(\boldsymbol{\xi}^{s}\right)=\nabla f^{i}\left(\boldsymbol{\xi}^{s}\right) \cdot \boldsymbol{S}_{k}^{i}\left(\boldsymbol{\xi}^{s}\right)
$$

Sampling $\Gamma_{k}^{i}$ over the evaluation points $\boldsymbol{\xi}_{n}^{s}$ is recast in a matrix format

$$
\left[\boldsymbol{G}^{s i}\right]_{n k}=\Gamma_{k}^{i}\left(\boldsymbol{\xi}_{n}^{s}\right)
$$

and similarly for the residuals

$$
\left\{\boldsymbol{\tau}^{s i}\right\}_{n}=\bar{\tau}^{i}\left(\boldsymbol{\xi}_{n}^{s}\right)
$$

that allows to express the $(s, i)$ linear system as

$$
\left[\boldsymbol{C}^{s i}\right]=\left[\boldsymbol{G}^{s i}\right]^{\dagger}\left[\boldsymbol{G}^{s i}\right]
$$

(the matrix multiplication consists of summing over evaluation points) and

$$
\left\{\boldsymbol{d}^{s i}\right\}=\left[\boldsymbol{G}^{s i}\right]^{\dagger}\left\{\boldsymbol{\tau}^{s i}\right\}
$$

The notation $[\boldsymbol{G}]^{\dagger}$ refers to the transposed of matrix $[\boldsymbol{G}]$.

For the first formulation using camera pairs, the spirit is the same but the writing appears less transparent. Using the previously introduced notation as block matrices

$$
\left[\boldsymbol{G}^{s i j}\right]_{n k}=\left[\begin{array}{ll}
{\left[\boldsymbol{G}^{s i}\right]} & -\left[\boldsymbol{G}^{s j}\right]
\end{array}\right]
$$

and the sampled residual

$$
\left\{\boldsymbol{\theta}^{s i j}\right\}_{n}=\bar{\theta}^{i j}\left(\boldsymbol{\xi}_{n}^{s}\right)
$$


give the final expression of matrix

$$
\left[\boldsymbol{C}^{s i j}\right]=\left[\boldsymbol{G}^{s i j}\right]^{\dagger}\left[\boldsymbol{G}^{s i j}\right]
$$

and second member

$$
\left\{\boldsymbol{d}^{s i j}\right\}=\left[\boldsymbol{G}^{s i j}\right]^{\dagger}\left\{\boldsymbol{\theta}^{s i j}\right\}
$$

In the above expression, the gray level corrections are not accounted for. They can be treated separately, first correcting for the motion, and then for the gray levels (and in this case, the determination of $\alpha$ is nothing but a least squares fit over polynomials). However because of the coupling between gray level corrections and displacements, it may be advantageous in terms of convergence rate to include $\alpha$ in the set of unknowns, using an augmented linear system [22]. There is no difficulty in writing this linear system, but the symbolic notations become prohibitively lengthy, and details are not provided here. This type of correction has been implemented herein. 